\title{
The "Molecular and Cell Biology" Program of the Presidium of the Russian Academy of Sciences as an Effective Format for the Support of Promising Scientific Research Groups
}

\author{
V.V. Sychev
}

There are various ways to finance science in Russia, both governmental and private. Financial support can range from tens of thousands of rubles up to several million in stipends and grants. One of the questions most often addressed to the heads of agencies or funds is about the level of transparency and objectivity when selecting groups which receive financial support. Few well-known financing organizations have avoided criticism regarding this issue.

Nevertheless, there is one scientific financing program that has earned the right to be called one of the most transparent and objective programs of its kind. This is the "Molecular and Cell Biology" program of the Presidium of the Russian Academy of Sciences (RAS), which is commonly named the Georgiev program after its coordinator, RAS academy member Georgiy Georgiev. According to numerous specialists, this is the best program in our country.

It has been 8 years since the Presidium of the RAS decided to launch an experimental program for awarding the most promising research groups with large sums of money. The idea behind this plan was to support leading scientific groups and bring the working conditions of these groups closer to international laboratory standards. The plan was to finance specific laboratories and not whole institutes, because it was clear that each institute had laboratories that differ in their success rates. Therefore, it was prudent to finance specialists which have proven their high qualifications. There was another factor in support of such an experiment. Science receives much more financial support in the United States and Western Europe than in Russia, which means that, in order to keep the scientific potential and talented (especially young) minds in the country, scientists must be offered financially comfortable conditions. It was suggested that the principal investigators could decide if their priorities were selectively high wages or reagents and equipment. Where should this new system of scientific financial support begin? The founders of the program had a clear understanding that they needed to avoid repeating the situation with the planned budget financing of research institutes. First of all, the division of finances between different organizations was somewhat arbitrary. Secondly, after the money was received by the granted organization, it was completely controlled by the administration. Both of these circumstances were considered unacceptable. However, the key issue was the same as in every competition. How and by whom would the selection of worthy research groups be accomplished? Various fields of science have fairly objective criteria for such a selective process, which are scientometric parameters: the impactfactor (IF) of the journal in which the author publishes his or her articles and the citation index (CI). The citation index shows how often a certain article is referenced by other researchers, thus quantifying the informational consequences this article has achieved. Of course this index is influenced by many random factors and it often biases the real value of an article. The impact factor shows how often an article published in a certain journal is cited annually, thus establishing a hierarchy of scientific journals. The higher the IF of a journal, the stricter the requirements towards the articles to be published in it are, thus making it harder to get published and increasing the estimated value of a work published in such a journal. Strict reviewing of articles in such 
journals is, for all practical purposes, an international expert committee for the evaluation of scientific merit, which is a very actively discussed topic. One of the fields where scientometric indices can be used as an objective indicator of the "promise" of a certain author or group is physico-chemical biology, which is a major part of modern life sciences. This is why the RAS Presidium created a special program in order to support scientists in this field. The program was initially called "Physico-Chemical Biology" (the name was changed in 2006 to "Molecular and Cell Biology" (MCB)), and RAS member Georgiy Georgiev became its coordinator. "We have three types of competitions" explained
Georgiy Georgiev on the principles of finance distribution. "One competition is strictly for fundamental research, another is for socially oriented projects. These are aimed at solving important practical problems, but the final result remains unpredictable, as in any fundamental research project. Finally, the third competition is for the support of so-called new groups." The award for new group is a special part of the program. Commonly, a certain bright and productive young researcher emerges from a laboratory and makes a bid for independence and for the fulfillment of his own projects and ideas. Such a researcher can make arrangements with the administration of any RAS insti- tute to provide him with a work space and a slot (slots) if he wins the MCB grant. In this case we mean supporting the work-force reserve (or life science "growth points" so to speak). This type of competition is also available to our fellow countrymen who reside abroad, although they do not receive any preferential treatment. The program also requires the head of the project to spend no less than 9 months of the year in a Russian laboratory, which practically means that the scientist in question must repatriate.

The amount of funds granted to specific projects varies depending on the type of competition. Until recently, fundamental and fundamentally-oriented

\section{SPECIALIST COMMENTARY}

\section{Arseniy Kaprelyants: "The unique thing about the Georgiev Program is its openness"}

Kaprelyants Arseniy Sumbatovich is a PhD in Biology, Head of the Microorganism Stress Biochemistry laboratory in the Bach Biochemistry Institute, RAS, and head of the project "Metabolic Pathways of Latent Tuberculosis Reactivation."

The program coordinated by RAS academy member Georgiy Georgiev is unique. There are no other programs like it. Its uniqueness is foremost the objectivity and transparency of the expert assessment. Of course other programs have expert assessment procedures, but they lack transparency. The applicant does not know the reasons for which his grant request has been denied, while another person wins the funding.

The "Molecular and Cell Biology" program is formalized, but you know the rules of the game. In my opinion, this is the correct approach in our current circumstances. In our country, when there is no objective mechanism of bid selection, all sorts of conflicts which have nothing to do with science begin to crop up, as well as other subjective factors.

In your opinion, is the know-how of the Georgiev program useful for the support of other fields of scientific research?

Yes, I think that in the current circumstances in Russia it would be advantageous. Many features of the expert assessment could be used in other RAS programs. They might not solve all the problems, but openness and transparency must certainly be incorporated.

What are your main achievements? What have you accomplished with the help of funding from the RAS Presidium's "Molecular and Cell Biology" program?

Even before we took part in this program, we discovered the resuscitation promoting factor (RPF) protein family in collaboration with English scientists from Wales University. These proteins are secreted by various bacteria (including mycobacteria, which cause tuberculosis). This awakens hibernating bacteria and induces them to shift from latency to active functioning. This was a very important discovery and we decided to continue this line of research. When we discovered these proteins, we did not know anything about them, how they worked or the mechanisms through which they functioned.

Using the program's funding we made one of our most important discoveries. We found that these proteins were enzymes that cleave specific molecules in the bacterial cell wall. In fact, we are very near to understanding how these proteins really work. We had a certain idea. We knew that the latent bacteria had increased cell-wall thickness, similarly to sporogenous bacteria. It seems that the RPF proteins cleave some type of bonds in the cell wall, thus softening it, which triggers active division. Before this, the hard outer "shell" prevented the cell from dividing. Thanks to this grant we have documented this mechanism and published the result in public journals.

The second achievement was the experimental proof of the importance of RPF proteins in the tuberculosis development in laboratory animals. In collaboration with our colleagues from South Africa, we studied the behavior of

M. tuberculosis strains with inactivated RPF genes (five genes). Knocking out no less than three of the five RPF genes lowered the virulence of $M$. tuberculosis strains in the tested animals. Moreover, we discovered that these strains could not be revitalized from their latent condition in vitro. Inactivating four RPF genes caused an almost complete loss of virulence in these strains. This lead to the idea that this strain could be used as a vaccine, since an avirulent strain presents no danger but the immune response could be strong. We could produce this strain in laboratory conditions, in a test tube, and then try to vaccinate laboratory animals with it. Which is what we did, with the help of our colleagues from the Central Scientific Research Institute of Tuberculosis at the Russian Academy of Medical Sciences (RAMS).

It turned out that these strains really do have a strong protective effect. Firstly, we vaccinated mice and then infected them with active tuberculosis. And most of the mice survived! Which meant that we had a high-efficiency vaccine that is no worse and maybe even better than the well-known Callmete-Guerin vaccine. Thus, the funding of our work by the Georgiev grant resulted in a very promising line of research.

Anna Gorbatova, STRF.ru, for Acta Naturae 


\section{Yulia Kravchenko: "I could feel that it is really possible to do science in Russia."}

Kravchenko Yulia Evgenyevna, PhD in Biology, head of the Genome Translation Regulation group at the Engelhardt Institute of Molecular Biology, RAS, and head of the project "A Study of the Functions of the Nuclear RNA-polymerase spRNAP-IV and Systems Providing Genome Stability" (new group).

Yulia Evgenyevna, how was this group created and what results have you obtained using funding from the "Molecular and Cell Biology" program?

The basis for the creation of this new group was the discovery of a new fourth nuclear RNA-polymerase in eukaryotes (the so-called spRNAP-IV, from the English single-polypeptide RNA polymerase IV). This was the subject of my doctorate thesis, which I successfully completed in 2004; in the following year I applied for the "New Groups" competition of the MCB program and received a grant for the development of a new scientific group.

The main theme of our group's research is the study of transcriptional processes. We need to find out which transcriptional factors are involved in the functioning of the new nuclear RNApolymerase, what the precise structure of the transcriptional complex is, which genes are transcribed by this polymerase, and which regulatory elements these genes use.

We have identified some components of the transcriptional complex. These are two of the three transcriptional factors of the mitochondrial RNA-polymerase. RNA-polymerase IV is an alternative splicing product of the POLRMT gene for the mitochondrial RNA-polymerase. We have demonstrated that two of the three transcriptional factors can be present in the nucleus; we also showed the direct interaction and complex formation between the novel polymerase and these factors. In other words, the mitochondrial RNA-polymerase and RNA-polymerase IV share these transcriptional factors. We are currently working on discovering the mechanism of transcription factor distribution: is it a competitive mechanism, or is it a switch from some proteins to others?

On the issue of which genes are transcribed by RNApolymerase IV, currently we have identified more than 50 of these genes. However, we cannot find any common function for these genes. Some of them are involved in metabolic pathways (such as glycolysis in mammals and yeast), others are involved in the remodeling of heterochromatin, DNA-reparation, conserving methylation status, encode heat-shock proteins, etc. Also, our data indicate that this new RNA-polymerase transcribes genes whose products are involved in signaling pathways, one of which is the signaling pathway of the p53 tumor suppressor protein. Thus, we can already assess the role of spRNAP-IV in the organism's life supporting processes just by analyzing the functions of spRNAP-IV-transcribed genes. Notably, we discovered a very high degree of similarity in the regulatory regions of all the identified genes. We found a functional motif in the promoters of all the studied genes, and this motif was not present in any of the promoters belonging to the genes which were transcribed by other RNA-polymerases. Notably, this unique conserved motif was found to be present in all mammals.

Another line of research conducted in our laboratory with support from the "Molecular and Cell Biology" program is the study of antioxidative protective mechanisms of the cellular genome. This work is done in collaboration with the Cellular Proliferation Laboratory headed by Petr Mikhailovich Chumakov.
Several years ago, Petr Mikhailovich's lab discovered the sestrin-2 protein, one of the three sestrin family proteins, which are activated by the $\mathrm{p} 53$ protein and which play a key role in the processes that protect the cell from excessive concentrations of active oxygen forms. Specifically, we demonstrated that sestrins act in conjunction with the sulphiredoxin protein to restore the oxidized forms of another component of the cellular oxidative protection system (the peroxiredoxin protein).

Until recently there has been no complex understanding of the functioning of the antioxidative protective system. There were only disjoined data on the functioning of separate proteins. However, we are the first to demonstrate the existence of a single multicomponent antioxidative protein complex, which includes sestrins, sulphiredoxin, thioredoxin reductase, and a number of other proteins which we have found to be a part of this structure. Interestingly, cells assemble this complex only in response to oxidative stress. While normally the components of the antioxidative complex are all localized in the cytoplasm, under stress the whole complex is transported into the nucleus, where it fulfills its main function: the protection of the genomic DNA from oxidation. We have also shown that this process is controlled by the $\mathrm{p} 53$ protein, which fulfills its "genome keeper" function, preventing damage to the DNA molecules.

How do you feel, being the head of a new scientific group?

After working for several years in the United States on a joint project and writing and completing my doctorate, I had the choice to find further work abroad or to stay here. To tell the truth, I didn't want to leave. Even though the work conditions for scientists abroad are great. Then I heard about the possibility of participating in the MCB program. I thought, OK, let's take a shot and apply, and I won a grant. The first year was mainly organization. We renovated the laboratory rooms which we got from the Institute using the funds from the program, bought equipment and reagents. As the head of the group, I had to delve into various organization issues, including the very peculiar way in which equipment and reagents would be bought in Russia. During this first and most difficult year, our group managed to slide on thanks to some earlier preliminary studies. On the other hand, I started feeling like you really could do science in Russia. I had a completely different outlook, much more optimistic. Moreover, being a part of the MCB program gives you the chance not only to work on scientific projects, but also to support people's way of life. MCB grants allow you to pay your workers a large enough salary that they do not need additional income. This creates fairly comfortable conditions for scientific creativity. Apart from myself, my group includes a $\mathrm{PhD}$ (who completed his doctorate during the work of this new group), three postgraduate students, and two undergraduates. Apart from that, I regularly receive letters from students asking if I take on postgraduates or undergraduates to complete their diploma project. Currently, I am thinking about taking on another undergraduate student; we have an interesting project. So this program is a good start for young and ambitious specialists. In addition, it is a good stimulus for a future career, since the work rate under the MCB program does not allow you to sit back and relax. The fact that you have to conform with the requirements of the Program gives you strength and keeps you on your toes (this mainly means obtaining results which are published in prestigious journals). However, the main motivating factor is still scientific curiosity, since without that you cannot be successful.

V. Sychev 
projects received up to 4 million rubles annually over the course of 5 years (although if the project is purely theoretical, the amount of allocated funds is, of course, considerably less). New groups could receive up to 2 million annually over the course of 3 years. "Of course these sums seemed rather good 7 years ago, but currently they call for doubling due to constant inflation," says Georgiy Georgiev. Instead, the financing of this program decreased in 2010 by practically a third (29\%). Accordingly, the sums allocated for each of the three competitions decreased. This immediately put the program on the verge of collapse, since its main goal, the support of promising research groups, was lost. This immediately initiated a new cycle of emigrations of young postdoctoral workers for a number of good laboratories.

How are the winning bids selected? A Scientific committee is formed by actively working members of the academy. The goal of this committee is to approve the general rules of bid selection. The same committee elects an Expert Committee consisting of seven members who organize the competition but whose role in the selection is minimal. The program must be automated to the largest possible extent and must not depend on the personal preferences of the coordinator or the overseers. The initial stage of any MCB competition is the accumulation of bids and their processing by technical groups of $2--3$ people from various institutes. This stage results in data summary sheets which demonstrate the objective indices of the laboratory's effectiveness or (in the case of new groups) the effectiveness of a single scientist. A lot of value is set on the sum of the IF of articles published in the last 5 years, factoring in the input of the laboratory into each of the publications and the IF factor per member of the group.

Nonscientometric indices are also used when assessing applications and the applicants must conform to these requirements. These include signs of acknowledgement by the Russian and international scientific community (various awards and titles), the age of the members of the project group (special attention is paid to young people and middle-aged researchers under 45). The training of workers in the group and patented works are also taken into account.

The summary data sheets are published on the program's website (each applicant can verify the data). Bids which show especially high objective indices (most of all, the impact factor) are automatically declared winners (these are usually about a third of the overall number of winners). The Expert Committee's task is to verify whether any violations took place in this process (for instance, if members of a group which are in fact emigrants were included in the publication count). Notably, bids from members of the Expert Committee cannot be winners in the first round, so as to prevent any question of their objective assessment.

The winners of the first round and the best (highest scoring) winners of previous competitions form the "top pool," which then conducts an independent expert assessment. Each of the remaining bids receives five independent reviews from well-known strong scientists in the field which have no personal interest in the scores of the bid in question.

"This 'top pool' reviews all the bids that did not win in the first round and also the bids from members of the Expert Committee," explains Georgiy Georgiev. "Experts can slightly change the scores based on objective reasons. For instance we increased the score for a person who had very good science but very poor knowledge of the English language. He had difficulties with being published abroad, but experts knew the real value of his work, and thus increased the score. Another example might be a person who works abroad but who already has no firm ties with Russia and who does not benefit Russian science in any way. But he is still nominally a member of a laboratory in our country and his publications are used to calculate the score of a bidding laboratory. Our experts watch out for such instances."

Bids for socially oriented projects are assessed with even more attention. The scores include not only the "strength" of the group, but also the scope (significance) and originality of the project and its potential for being fulfilled. After the expert assessment, these indices are added to the objective criteria. The Expert Committee then makes its final decision, which can vary only slightly from the objective scores and expert recommendations.

The results of the scoring procedure and the decisions are published at once, and the author can view the expert opinions (upon request) and appeal to the Expert Committee or the specially formed Controlling Committee, which consists of academy members who do not work in the RAS system or do not work in its Central Region. In several cases the initial decisions had to be reversed.

"How are the funds distributed? Usually our program has a limit in terms of the amount of funds available for wages; this conforms to general academic expenditures." says Gerogiy Georgiev. "In 2010 we were told that this sum should be about $50-55 \%$ of the overall funds. The specifics are decided by the head of the group. The head of the laboratory and the staff are all responsible for the result; if there are no publications in 5 years or if the group violates the rules of the program, funding will cease."

"Molecular and Cell Biology" has already proven its worth. The success of 7 years of work is indirectly confirmed by the fact that almost all of the research groups supported by its funding are top specialists in life sciences in Russia. Of course the distribution of funds in the "Molecular and cell biology" program can be discussed, but a vast majority of specialists in our country acknowledge that no other program does a better job. It would be a very good thing if other scientific fields in Russia were to implement similar ways to distribute funding. 